\title{
A Simple Approach for Finding a Fair Solution to Multiobjective Programming Problems
}

\author{
P. Pandian
}

\author{
Department of Mathematics, School of Advanced Sciencs, VIT University, Vellore-14, Tamil Nadu, \\ India
}

\begin{abstract}
Keywords: Multiobjective programming problem, Efficient solution, Fair solution, Sum of objectives method
\end{abstract}

\begin{abstract}
A new approach, namely sum of objectives (SO) method is proposed to finding a fair solution to multi-objective programming problems. The proposed method is very simple, easy to use and understand and also, common approaches. It is illustrated with the help of numerical examples. The fair solution serves more better than efficient solution for decision makers when they are handling multiple objective decision making problems.
\end{abstract}

Mathematics subject classification: 65K05, 90C29.

\section{Introduction}

Multi objective optimization is the process of simultaneously optimizing two or more conflicting objectives subject to certain constraints. Such problems can arise in practically every field of Science, Engineering and Management Science. Due to the conflicting nature of the objectives, a unique feasible solution optimizing all the objectives does not exist. Pareto [6] introduced an efficient solution to a multiobjecitve programming (MOP) problem. In the literature, a variety of algorithms $[1,9,8,5,2,4]$ have been proposed to find a set of efficient solutions to the MOP problem. For getting good solution from a set of efficient solutions, a decision maker is needed to provide additional preference information and to identify the most satisfactory solution. Efficient solutions may be classified into two categories, namely improper and proper. Geoffrion [3] introduced a scaling method to find a properly efficient solution to the MOP problem. An improperly efficient solution to the MOP problem is extremely biased, but the properly efficient solution is a moderately good. Multiobjecitve decision making (MODM) refers to the solving of decision problems involving multiple and conflicting objectives, coming up with a final solution that represents a good compromise that is acceptable to the entire team. Therefore, a properly efficient solution is best option for MODM problems.

In this paper, we propose a new method namely, sum of objectives (S0) method for finding a properly efficient solution ( a fair solution ) to MOP problems. The proposed method is very simple, easy to use and understand and also, common approaches. It provides a useful tool for solving MODM problems. The proposed method is illustrated with the help of numerical examples. The fair solution is an unbiased solution and also, serves more better than efficient solution for decision makers when they are handling multiple objectives without providing additional preference information.

\section{Preliminaries}

Consider the following multi-objective mathematical programming problem

(P) Minimize $f(x)=\left(f_{1}(x), f_{2}(x), \ldots, f_{k}(x)\right)$

$$
\text { subject to } g(x) \leqq 0, \quad x \in X,
$$

where $f_{\mathrm{i}}: X \rightarrow R, \mathrm{i}=1,2, \ldots, \mathrm{k}$ and $g: X \rightarrow R^{\mathrm{m}}$ where $g=\left(g_{1}, \ldots, g_{m}\right)$ are differentiable functions on $X$, an open convex subset of $R^{\mathrm{n}}$.

Let $\mathrm{P}=\left\{x \in X: g_{\mathrm{j}}(x) \leq 0, \mathrm{j}=1,2, \ldots m\right\}$. That is, $\mathrm{P}$ is the set of all feasible solutions for the problem (P). 
Definition 2.1: A feasible point $x^{\circ}$ is said to be efficient for $(\mathrm{P})$ if there exists no other feasible point $x$ in $(\mathrm{P})$ such that $f_{\mathrm{i}}(x) \leq f_{\mathrm{i}}\left(x^{\circ}\right), \mathrm{i}=1,2, \ldots, \mathrm{k}$ and $f_{\mathrm{r}}(x)<f_{\mathrm{r}}\left(x^{\circ}\right)$, for some $\mathrm{r} \in\{1,2, \ldots, \mathrm{k}\}$.

Definition 2.2: An efficient solution $x^{\circ}$ is said to be a fair (properly efficient ) solution of (P) if there exists a scalar $\mathrm{M}>0$ such that, for each $\mathrm{i} \in\{1,2, \ldots, \mathrm{k}\}$ and for all feasible $x$ of $(\mathrm{P})$ satisfying $f_{\mathrm{i}}(x)<f_{\mathrm{i}}\left(x^{\circ}\right)$, we have

$$
f_{\mathrm{i}}\left(x^{\circ}\right)-f_{\mathrm{i}}(x) \leq M\left(f_{\mathrm{r}}(x)-f_{\mathrm{r}}\left(x^{\circ}\right)\right)
$$

for some r such that $f_{\mathrm{r}}(x)>f_{\mathrm{r}}\left(x^{\circ}\right)$.

A solution of $(\mathrm{P})$ is said be improper efficient solution of $(\mathrm{P})$ if it is efficient, but not properly efficient.

\subsection{Importance of Fair ( properly efficient) Solution}

Suppose that $x^{\circ}$ is an improper efficient solution of $(\mathrm{P})$. Then, for any $\mathrm{M}>0$, there is an $\mathrm{i} \in\{1,2, \ldots, \mathrm{k}\}$ and $x \in \mathrm{P}$ with $f_{\mathrm{i}}(x)>f_{\mathrm{i}}\left(x^{\circ}\right)$ such that

$$
f_{\mathrm{i}}(x)-f_{\mathrm{i}}\left(x^{\circ}\right)>M\left[f_{\mathrm{r}}\left(x^{\circ}\right)-f_{\mathrm{r}}(x)\right] \text { for all } \mathrm{r} \text { with } f_{\mathrm{r}}\left(x^{\circ}\right)>f_{\mathrm{r}}(x) .
$$

By letting $\mathrm{M} \rightarrow \infty$, this implies the existence of some objective $i^{*}$ whose marginal gain can be made arbitrarily large relative to each of the marginal losses in all other objectives. This is an extreme unfairness of an improper efficient solution of (P). This is the main weakness of an improper efficient solution.

Therefore, the properly efficient solution of the problem $(\mathrm{P})$, called fair solution of the problem (P) is a moderately good, unbiased and also, serves more better than efficient solution for decision makers when they are handling multiple decision making problems in general manner.

We need the following result which can be found in [2].

Theorem 2.1: Let $\lambda^{\circ}>0$ in $R^{k}$ be fixed with $\lambda^{\circ T} \mathrm{e}=1$. If $x^{\circ}$ is an optimal solution of the scalar programming problem $\left(P_{\lambda^{\circ}}\right)$ where

$\left(P_{\lambda^{\circ}}\right)$ Minimize $\lambda^{\circ T} f(x), x \in \mathrm{P}$,

then $x^{\circ}$ is a fair solution of the problem (P).

\section{Main Results}

Now, we establish a result between a fair solution of a MOP problem and an optimal solution of its scalarization problem.

Lemma 3.1: Let $\lambda^{\circ}>0$ in $R^{k}$ be fixed with $\lambda^{\circ T} \mathrm{e}=1$ and $\mathrm{K}$ be a positive fixed constant. $x^{\circ}$ is an optimal solution of the scalar programming problem $\left(P_{\lambda^{\circ}}\right)$ where

$$
\left(P_{\lambda^{\circ}}\right) \quad \text { Minimize } \lambda^{\circ T} f(x), \quad x \in \mathrm{P},
$$

iff $x^{\circ}$ is an optimal solution of the scalar programming problem $\left(Q_{\lambda^{\circ}}\right)$ where

$$
\left(Q_{\lambda^{\circ}}\right) \text { Minimize } \mathrm{K} \lambda^{\circ T} f(x), x \in \mathrm{P} .
$$

Proof: Now, since $\mathrm{K}$ is a positive fixed constant, we have for any $\mathrm{x} \in \mathrm{P}$,

$$
\lambda^{\circ T} f\left(x^{\circ}\right) \leq \lambda^{\circ T} f(x) \Leftrightarrow K \lambda^{\circ T} f\left(x^{\circ}\right) \leq K \lambda^{\circ T} f(x) .
$$

Hence the theorem. 
Theorem 3.2: If $x^{0}$ is an optimal solution of the scalar programming problem (S) where

$$
\text { (S) Minimize } \sum_{\mathrm{i}=1}^{\mathrm{k}} f_{\mathrm{i}}(x), \quad x \in \mathrm{P} \text {, }
$$

then $\mathrm{x}^{\mathrm{o}}$ is a fair solution of the problem $(\mathrm{P})$.

Proof: Taking $\lambda_{\mathrm{i}}^{\circ}=\frac{1}{\mathrm{k}}, \mathrm{i}=1,2, \ldots, \mathrm{k}$ and $\mathrm{K}=\mathrm{k}$.

Now, $K \lambda^{\circ T} f(x)=\sum_{\mathrm{i}=1}^{\mathrm{k}} f_{\mathrm{i}}(x)$.

Therefore, $\mathrm{x}^{\mathrm{o}}$ is an optimal solution of the scalar programming problem $\left(Q_{\lambda^{\circ}}\right)$ where

$$
\left(Q_{\lambda^{\circ}}\right) \quad \text { Minimize } K \lambda^{\circ T} f(x), \quad x \in \mathrm{P} .
$$

By the lemma 3.1., we can conclude that $\mathrm{x}^{\mathrm{o}}$ is an optimal solution of the scalar programming problem $\left(P_{\lambda^{\circ}}\right)$ where

$$
\left(P_{\lambda^{\circ}}\right) \quad \text { Minimize } \lambda^{\circ T} f(x), x \in \mathrm{P} .
$$

By the Theorem 2.1., we can conclude that $\mathrm{x}^{\mathrm{o}}$ is a fair solution of the problem(P) .

Hence the theorem.

Now, we introduce a new method namely, sum of objectives method for finding a fair solution to multi-objective optimization problems.

Algorithm:

Step 1: Write the single objective optimization problem (S) for the given multi-objective optimization problem.

Step 2: Solve the problem (S) using any known optimization method. Let $\mathrm{x}^{\mathrm{o}}$ be an optimal solution for the problem (S).

Step 3: $\mathrm{x}^{\mathrm{o}}$ is a fair solution for the problem (P) ( by the Theorem 3.2.).

\section{Numerical Examples}

The proposed method is illustrated by the following numerical examples.

Example 4.1: Consider the following multi-objective nonlinear programming problem

(P) Minimize $\left(f_{1}(x, y, z), f_{2}(x, y, z), f_{3}(x, y, z)\right)$

$$
=\left(x^{2}+4 y+3 z-20,10 x+y^{2}+3 z-30, x^{2}+4 y+3 z^{2}-50\right)
$$

subject to $x+y+z=20 ; x, y, z \geq 0$.

The problem $(\mathrm{S})$ is given below

(S) Minimize $w=2 x^{2}+y^{2}+3 z^{2}+10 x+8 y+6 z-100$

Subject to $x+y+z=20 ; x, y, z \geq 0$.

The optimal solution of the problem (S) by the method of Lagrangian multipliers is $x=5, y=11, z=4$.

Therefore, the fair solution of (P) is $x=5, y=11, z=4$ and the objective value is $(61,153,47)$ 
Example 4.2: Consider the following multi-objective linear programming problem

(P) Maximize $z=\left(4 x_{1}+20 x_{2}+4 x_{3}, 2 x_{1}-2 x_{2}+5 x_{3}, 6 x_{1}-3 x_{2}+5 x_{3}\right)$

Subject to

$$
-x_{1}+x_{2} \leq 0 ;-x_{2}+2 x_{3} \leq 0 ; x_{1}+x_{2}+x_{3} \leq 100
$$$$
x_{1}, x_{2}, x_{3} \geq 0 \text {. }
$$

The optimal solution of the problem (S) by simplex method is $\left(x_{1}, x_{2}, x_{3}\right)=(40,40,20)$.

Therefore, a fair solution of the problem (P) is $\left(x_{1}, x_{2}, x_{3}\right)=(40,40,20)$ and the objective value is $(320,100,260)$.

Example 4.3: Consider the following multi-objective integer linear programming problem

(P) Maximize $z=\left(x_{1}+x_{2}+x_{3},-2 x_{1}+x_{2}+x_{3}, 3 x_{1}-x_{2}+x_{3}\right)$

subject to

$$
\begin{aligned}
& x_{1}+2 x_{2}+x_{3} \leq 6 ; 2 x_{1}+x_{2}-x_{3} \leq 4 ; 2 x_{2}+3 x_{3} \leq 6 \\
& x_{1}, x_{2}, x_{3} \geq 0 \text { and are integers. }
\end{aligned}
$$

The problem $(\mathrm{S})$ is given below

(S) Maximize $w=2 x_{1}+x_{2}+3 x_{3}$

subject

$$
\begin{aligned}
& x_{1}+2 x_{2}+x_{3} \leq 6 ; 2 x_{1}+x_{2}-x_{3} \leq 4 ; 2 x_{2}+3 x_{3} \leq 6 \\
& x_{1}, x_{2}, x_{3} \geq 0 \text { and are integers. }
\end{aligned}
$$

The optimal solution of the problem (S) by branch and bound method is $\left(x_{1}, x_{2}, x_{3}\right)=(3,0,2)$. Therefore, a fair solution of the problem $(\mathrm{P})$ is $\left(x_{1}, x_{2}, x_{3}\right)=(3,0,2)$ and the objective value $=(5,-$ $8,11)$.

Example 4.4. Consider the following bi-criteria transportation problem

\begin{tabular}{|l|l|l|l|l|l|}
\hline & & & & & Supply \\
\hline & $(1,4)$ & $(2,4)$ & $(7,3)$ & $(7,4)$ & 8 \\
\hline & $(1,5)$ & $(9,8)$ & $(3,9)$ & $(4,10)$ & 19 \\
\hline & $(8,6)$ & $(9,2)$ & $(4,5)$ & $(6,1)$ & 17 \\
\hline Demand & 11 & 3 & 14 & 16 & \\
\hline
\end{tabular}

The problem $(\mathrm{S})$ is given below

\begin{tabular}{|l|l|l|l|l|l|}
\hline & & & & & Supply \\
\hline & 5 & 6 & 10 & 11 & 8 \\
\hline & 6 & 17 & 11 & 14 & 19 \\
\hline & 14 & 11 & 9 & 7 & 17 \\
\hline Demand & 11 & 3 & 14 & 16 & \\
\hline
\end{tabular}

Now, by the zero point method [7], the optimum solution of the problem (S) is given below:

\begin{tabular}{|l|l|l|l|l|l|}
\hline & & & & & Supply \\
\hline & $\mathbf{5}-\theta$ & $\mathbf{3}$ & $\theta$ & & 8 \\
\hline & $\mathbf{6}+\theta$ & & $\mathbf{1 3 -} \theta$ & & 19 \\
\hline & & & $\mathbf{1}$ & $\mathbf{1 6}$ & 17 \\
\hline Demand & 11 & 3 & 14 & 16 & \\
\hline
\end{tabular}

The problem has six solutions $x_{11}=5-\theta ; \quad x_{12}=3 ; x_{13}=\theta ; x_{21}=6-\theta ; x_{23}=13-\theta ; x_{33}=1 ;$ $x_{34}=16$ and $(176-\theta, 175+\theta)$ where $\theta=\{0,1,2,3,4,5\}$ 
Therefore, a fair solution of the problem $(\mathrm{P})$ is given below

\begin{tabular}{|l|l|l|r|r|l|}
\hline & & & & & Supply \\
\hline & $(1,4)$ & $(2,4)$ & $(7,3)$ & $(7,4)$ & 8 \\
& $\mathbf{2}$ & $\mathbf{3}$ & $\mathbf{3}$ & & \\
\hline & $(1,5)$ & $(9,8)$ & $(3,9)$ & $(4,10)$ & 19 \\
$\mathbf{9}$ & & $\mathbf{1 0}$ & & \\
\hline & $(8,6)$ & $(9,2)$ & $(4,5)$ & $(6,1)$ & 17 \\
& & & $\mathbf{1}$ & $\mathbf{1 6}$ & \\
\hline Demand & 11 & 3 & 14 & 16 & \\
\hline
\end{tabular}

and the fair total transportation cost is $(168,185)$.

Remark 4.1: The fair solution is better than the optimal compromise solution $\mathrm{x}_{12}=3 ; \mathrm{x}_{13}=2 ; \mathrm{x}_{14}=$ $3 ; \mathrm{x}_{21}=11 ; \mathrm{x}_{23}=8 ; \mathrm{x}_{33}=4 ; \mathrm{x}_{34}=13$ and the objective function value $=(170,190)$ in [10].

\section{Conclusion}

The new method namely, sum of objectives (SO) method for finding a properly efficient solution (a fair solution ) to MOP problems is introduced. The proposed method is very simple, easy to use and understand and also, common approaches. It is unbiased and provides more moderate solution to MOP problems. The SO method will be served a suitable tool for solving MODM problems since it provides a fair solution to MOP problems.

\section{References}

[1] V. Chankong and Y.Y. Haimes, Multi-objective Decision Making Theory and Methodology, North-Holland, New York, 1983.

[2] Ebrahim A. Youness, A direct approach for finding all efficient solutions for multiobjective programming problems, European Journal of Operational Research, 81, 1995, 440-443.

[3] A.M.Goeffrion, Proper efficiency and the theory of vector maximization, J. Math. Anal. Appl., 22, 1968, 618-630.

[4] R.T. Marler and J.S. Arora, Survey of multi-objective optimization methods for engineering, Struct. Multidisc. Optim., 26, 2004, 369-395.

[5] K. Miettinen, Nonlinear multi-objective optimization, Kluwer Academic Publishers, Boston, 1999.

[6] V.Pareto, Manuale di economica politica, Societa Editrice Libraria, Milan, 1906.

[7] P.Pandian and G.Natarajan, A new method for finding an optimal solution for transportation problems, Int. J. of Math. Sci. \& Engg. Appls., 4 , 2010, 59-65

[8] S. Ruzika and M. M. Wiecek, Approximation methods in multi-objective programming, Journal of Optimization Theory and Applications, 126, 2005, 473-501.

[9] T.L.Vincent and W.J. Grantham, Optimality in parametric systems, John Wiley and Sons, New York, 1981.

[10] Waiel F. Abd and El-Wahed, A multi-objective transportation problem under fuzziness, Fuzzy Sets and Systems, 117, 2001, 27-33. 